\title{
Comparison of the effect of insulin glargine and regular insulin on perioperative glycemic control in patients with type 2 diabetes mellitus under insulin treatment during vitrectomy surgery under general anesthesia: A randomized clinical trial study
}

\author{
Pooya Derakhshan ${ }^{1} * \mathbb{D}$, Ali Khatibi ${ }^{2}$, Seyed Hamid Reza Faiz ${ }^{1}$, Poupak Rahimzadeh ${ }^{1}$, Nasrin Nouri ${ }^{3}$ \\ Received: 25 Jul 2019 \\ Published: 6 Mar 2021
}

\begin{abstract}
Background: Perioperative glycemic control is an important factor in the clinical management of a patient with diabetes mellitus under surgery. Poorly controlled long-term hyperglycemia not only predisposes individuals to systemic complications of diabetes mellitus and cardiovascular morbidity but also increases the risk of anesthesia and weakens the outcome of the surgery. Given the importance of the issue and the limited studies on glucose control using insulin glargine during surgery, we aimed to investigate the effects of glargine on glucose control in patients with diabetes mellitus during vitrectomy surgery.

Methods: This randomized, double-blind trial was conducted in two groups of 35 patients with diabetes mellitus under treatment with insulin. In the control group, the patients received regular insulin based on the blood glucose and the sliding scale, and in the intervention group, they received insulin glargine $(0.3 \mathrm{unit} / \mathrm{kg})$ before surgery. From the start of the operation up to 3 hours of the surgery blood glucose of the patients was measured every 45 minutes and once 6 hours after the operation, and if needed, the regular insulin was injected. Data were analyzed using SPSS 16. Frequency, percentage, mean, and standard deviation (SD) were used to describe the data. To compare the quantitative variables, the independent t-test or U-Mann-Whitney test was used. For comparison of the qualitative variables, Chi-square test or Fischer's exact test and repeated measure ANOVA was employed. The significance level (P-value) was considered as $\mathrm{p}<0.05$.
\end{abstract}

Results: Use of insulin glargine was associated with significantly lower blood glucose levels compared to regular insulin at 90 minutes $(\mathrm{p}=0.004), 135$ minutes $(\mathrm{p}=0.001)$, and 6 hours after the operation $(\mathrm{p}=0.005)$.

Conclusion: Glycemic control using glargine compared to regular insulin has a better performance with less need for surplus insulin dose administration during surgery.

Keywords: Type 2 Diabetes Mellitus, Regular Insulin, Insulin Glargine, Perioperative Glycemic Control

\author{
Conflicts of Interest: None declared \\ Funding: None \\ *This work has been published under CC BY-NC-SA 1.0 license. \\ Copyright $\odot$ Iran University of Medical Sciences
}

Cite this article as: Derakhshan P, Khatibi A, Faiz SHR, Rahimzadeh P, Nouri N. Comparison of the effect of insulin glargine and regular insulin on perioperative glycemic control in patients with type 2 diabetes mellitus under insulin treatment during vitrectomy surgery under general anesthesia: A randomized clinical trial study. Med J Islam Repub Iran. 2021 (6 Mar);35:31. https://doi.org/10.47176/mjiri.35.31

Introduction

Currently, $11.3 \%$ of people over the age of 20 in the

Corresponding author: Dr Pooya Derakhshan, derakhshan.p@iums.ac.ir

1. Anesthesiology Department, Rasool Akram Hospital, Iran University of Medical Sciences, Tehran, Iran

2. Anesthesiology Department, Firoozgar Hospital, Iran University of Medical Sciences, Tehran, Iran

3. Anesthesiology Department, Fatima Plastic And Reconstructive Surgery Hospital, Iran University of Medical Sciences, Tehran, Iran
United States suf fer from diabetes mellitus (DM), and the

\section{$\uparrow$ What is "already known" in this topic:}

Hyperglycemia is accompaniedl by increased mortality risk, morbidity after major surgery, especially cardiac surgery, dehydration, electrolyte impairment, and delay in wound and infection healing, postoperative inflammation, hospital stay prolongation, cardiac complications, and acute renal failure after surgery.

\section{$\rightarrow$ What this article adds:}

Controlling the perioperative blood sugar using glargine was more efficient and with less need for an extra dosage of regular insulin during and after surgery, and it can be used to control the perioperative blood glucose levels as well. 
prevalence of this disease is on the rise. Based on the World Health Organization (WHO) estimates, there will be 300 million patients with DM by the year 2025 (1). Type $2 \mathrm{DM}$ is a systemic and progressive disease. The progressive nature of type $2 \mathrm{DM}$ means that patients need different types of treatment at different stages of the disease (Diet, exercise, oral hypoglycemic agents, and insulin therapy). Although Neutral Protamine Hagedorn (NPH) insulin is the basis for basal replacement therapy, it requires several daily doses due to the length of effect of fewer than 24 hours and is associated with a higher risk of overnight hypoglycemia compared to glargine due to unwanted peaks in plasma insulin. The objective of exogenous insulin is to control blood glucose and maintain glycated hemoglobin (HbA1c), fasting plasma glucose (FPG) test, and postprandial plasma glucose (PPG) levels close to normal values to prevent or delay microvascular and macrovascular complications (2). Insulin glargine is a recombinant human insulin analog obtained through DNA technology using non-pathogenic Ecoli. This molecule is soluble in slightly acidic conditions $(\mathrm{pH}=4)$, which is why the absorption of this insulin is delayed and provides the base insulin requirement without a peak at insulin level for approximately 24 hours. The use of glargine is a once-daily subcutaneous injection that has been shown to control glucose levels in adults and children over 6 years of age with type $1 \mathrm{DM}$ and adults with type $2 \mathrm{DM}$. The low rate of glargine absorption results in a constant plasma level within 24 hours and the use of this insulin have been satisfactory in most of the patients with types 1 and 2 DM $(3,4)$.

Patients with DM usually suffer from major organ dysfunction due to systemic vascular disorders. Thus, in patients with DM, it seems that the rate of postoperative complications and poor prognosis are higher in comparison with patients without DM (5) . According to studies, patients with DM are more likely to undergo surgery, and $\mathrm{DM}$ is the most common endocrine disease anesthesiologist deals with $(6,7)$. Several observational and interventional studies have shown that hyperglycemia is accompanied by increased mortality risk, morbidity after major surgery, especially cardiac surgery, dehydration, electrolyte impairment and delay in wound and infection healing, postoperative inflammation, hospital stay prolongation, cardiac complications, and acute renal failure (secondary to increased oxidative stress in xanthine path and urinary excretion of inflammatory cytokine among patients with and without DM) after surgery (8-12). Van den et al. showed that accurate glycemic control in the range of 80$110 \mathrm{mg} / \mathrm{dl}$ was effective in reducing short-term mortality (30 days), sepsis reduction, surgery site infections, atrial fibrillation, and acute kidney injury after surgery, especially in cardiac surgery groups, albeit a higher chance of hypoglycemia $(<70 \mathrm{mg} / \mathrm{dl})(13)$.

Taking into account the importance of the issue and the limited studies on glycemic control using insulin glargine during surgery, and the lack of a similar study in Iran, we aimed to investigate the effect of glargine on glucose control in patients with DM during vitrectomy surgery.

\section{Methods}

This study was a randomized, double-blind controlled trial. The patients for the study were chosen from the patients with type $2 \mathrm{DM}$ under treatment with insulin who were referred to Rasool Akram Hospital, Tehran, Iran. The study inclusion criteria were: candidate for vitrectomy, aged 30-70 years, history of type 2 DM for more than 3 months, under insulin therapy, and New York heart association (NYHA) activity class 1 or 2. Similarly, the exclusion criteria included patient's unwillingness or lack of satisfaction as well as lack of adequate understanding of the study objective, a history of diabetic ketoacidosis, serum creatinine above $2 \mathrm{mg} / \mathrm{dL}$, hepatic failure, pregnancy, patients with mental disorders who could not provide information or consent, taking corticosteroids, infection and sepsis, body mass index $(\mathrm{BMI})>35 \mathrm{~kg} / \mathrm{m}^{2}$, fasting capillary blood glucose (FCG) below $150 \mathrm{mg} / \mathrm{dl}$ or above 300 $\mathrm{mg} / \mathrm{dl}$.

At the beginning of the study, written consent was obtained from all patients after giving complete explanations. The trial was registered in the ethics committee of Iran University of Medical Sciences with the code IR.IUMS.FMD.REC1396.9411174022 and in IRAN Clinical Registry with the code IRCT20141127020112N8 code.

The sample size was calculated using $\mathrm{G}^{*}$ Power, with an alpha of 0.05 and power of $80 \%$. Patients were randomly assigned to one of the groups receiving glargine (intervention) or receiving the regular insulin (control) to complete the sample volume using a computerized random number table.

All long-acting oral agents and insulin of the patient were stopped at night before surgery. On the morning of the operation day, the blood sample was checked for fasting blood glucose and recorded. On the entrance of the operating room, demographic information (age, sex), weight, height, body mass index(BMI), NYHA function class, associated illnesses, lab tests (creatinine, BUN, hemoglobin, sodium, potassium, and $\mathrm{HbA} 1 \mathrm{C}$ ) were entered into the prepared sheets and glucose of the eligible patients were checked with a glucometer (CHEK, TD-4230 LEVER) and according to the group number, at least two hours before the start of the operation, a number of the patients received insulin glargine (100 units/ml, Lantus solostar) as 0.3 units per kilogram of body weight subcutaneously. Moreover, the other group received regular insulin (HumulinN, Lily) subcutaneously at least 30 minutes before surgery based on capillary glucose and on the basis of the insulin protocol by sliding scale (14) and regardless of the amount of insulin used before the operation. The dosage of insulin was recorded and it was explained to the patients that insulin injections would be carried out in their arm, but the patient did not see the type of insulin syringe during the injection. Before the start of anesthesia, a capillary blood glucose sample was checked and recorded. After standard monitoring, patients underwent pre-oxygenation followed by general anesthesia with fentanyl $2 \mu \mathrm{g} / \mathrm{kg}$, midazolam $15 \mu \mathrm{g} / \mathrm{kg}$ of body weight, and as induction of propofol 1.2-2 $\mathrm{mg}$, and cisatracurium 
Table 1. Regular insulin protocol

\begin{tabular}{ccc}
\hline Blood Glucose level $(\mathrm{mg} / \mathrm{dl})$ & Human Insulin (IU) & Additional Action Requested \\
\hline $0-80$ & 0 & 1 Ampulle D50, Call a physician \\
$81-100$ & 0 & No action \\
$101-150$ & 0 & No action \\
$151-200$ & 2 & No action \\
$201-250$ & 4 & No action \\
$251-300$ & 6 & No action \\
$301-350$ & 8 & No action \\
$351-400$ & 10 & No action \\
$>401$ & 12 & Call physician \\
\hline
\end{tabular}

$0.2 \mathrm{mg} / \mathrm{kg}$ of body weight, and were intubated with the PVC endotracheal tube and received propofol maintenance as $100 \mu \mathrm{g} / \mathrm{kg}$ of body weight per minute. After the initiation of surgery, the capillary blood glucose, along with the vital signs, were checked and recorded every 45 minutes. In case of hypoglycemia or sweating, the blood glucose was checked again and in case of the values of under $70 \mathrm{mg} / \mathrm{dl}$, 50 milliliters infusion of $50 \%$ dextrose was administered for the patient, and in both groups, in the event of blood glucose levels above $200 \mathrm{mg} / \mathrm{dl}$, the patient received intravenous regular insulin as the following Table 1.

At the end of the surgery, a venous blood sample was taken to measure potassium, and patients were extubated by receiving neostigmine $0.04 \mathrm{mg} / \mathrm{kg}$ and atropine 0.02 $\mathrm{mg} / \mathrm{kg}$ of body weight, and transferred to the Postanesthesia Care Unit (PACU) and were monitored for hypoglycemia symptoms. It should be noted that the anesthesiologist that was responsible for collecting information and checking blood glucose and insulin injections was not aware of the patient group during anesthesia and surgery six hours after surgery, blood capillary glucose was measured and, if needed, insulin was injected. All measurements of blood glucose levels, the incidence of hypoglycemia, the required dose of regular insulin and the duration of surgery were inserted in the relevant sheets.

The collected data were analyzed using SPSS 16. Frequency, percentage, mean, and standard deviation (SD) were used to describe the data. To compare the quantitative variables, the independent t-test or U-Mann-Whitney test was used. For comparison of the qualitative variables, Chi-square test or Fischer's exact test and repeated measure ANOVA was employed. The significance level (Pvalue) was considered as $\mathrm{p}<0.05$ in all tests.

\section{Results}

The samples were randomly divided into two groups of 35 (Consort diagram is shown in Figure 1).

The mean age of the patients in the group receiving regular insulin was $58.77 \pm 10.28$ years, with the lowest and highest age in this group as 32 and 69 years, respectively. In addition, in patients receiving insulin glargine (Lan-
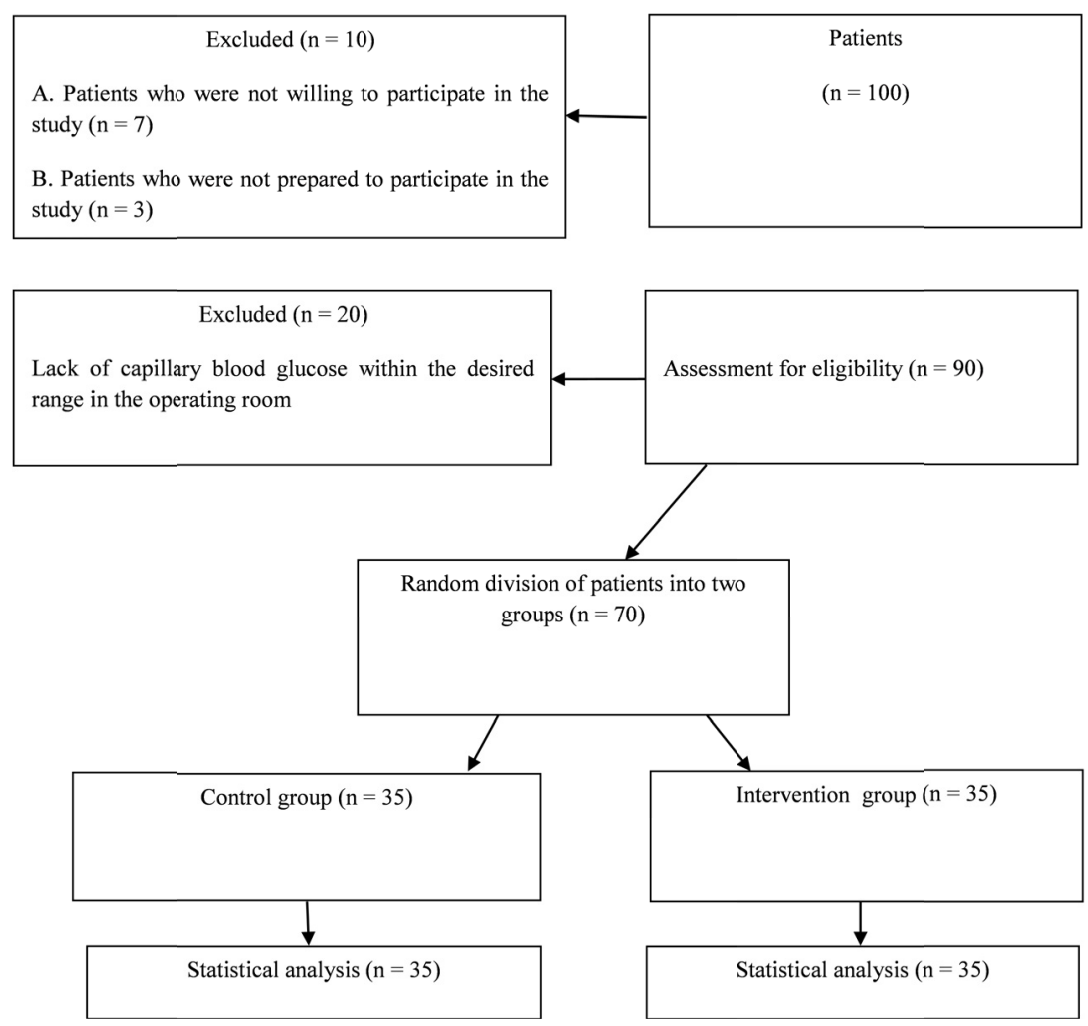

Fig. 1. Consort diagram 
thus), the mean age of the patients was $58.17 \pm 11.33$ years, with the highest and lowest age as respectively 70 and 38 years. There was no significant difference between the two groups in terms of age $(p=0.817)$.

Of the 35 patients in the regular insulin group, 22 $(62.9 \%)$ and $13(37.1 \%)$ were men and women, respectively. In patients of the insulin glargine (Lanthus) group, $19(54.3 \%)$ and $16(45.7 \%)$ patients were men and women, respectively, and there was no significant difference between the two groups $(\mathrm{p}=0.469)$ (Table 2$)$.

Table 3 demonstrates the experimental results of patients in the two groups. The duration of the surgery in the regular and glargine insulin groups was $3.18 \pm 0.40$ and $3.37 \pm 0.60$ hours, respectively $(\mathrm{p}=0.17)$. Table 3 indicates the mean blood glucose in patients before surgery (in the operating room), and at 45,90,135, and 180 minutes after starting surgery, and blood glucose of the patients 6 hours after surgery, which indicates that the blood glucose of patients at $90(\mathrm{p}=0.004)$ and 135 minutes after starting the surgery $(p=0.001)$ and 6 hours after the surgery $(p=0.005)$ were significantly different.

None of the patients in both regular and glargine (lanthus) insulin groups experienced hypoglycemia during surgery and 6 hours after the end of the surgery.

Tables 4 and 5 demonstrate the amount of regular insulin required in the patients in both groups at 45, 90, 135, and 180 minutes after starting the surgery and 6 hours after the end of the surgery. 6 hours after the end of the

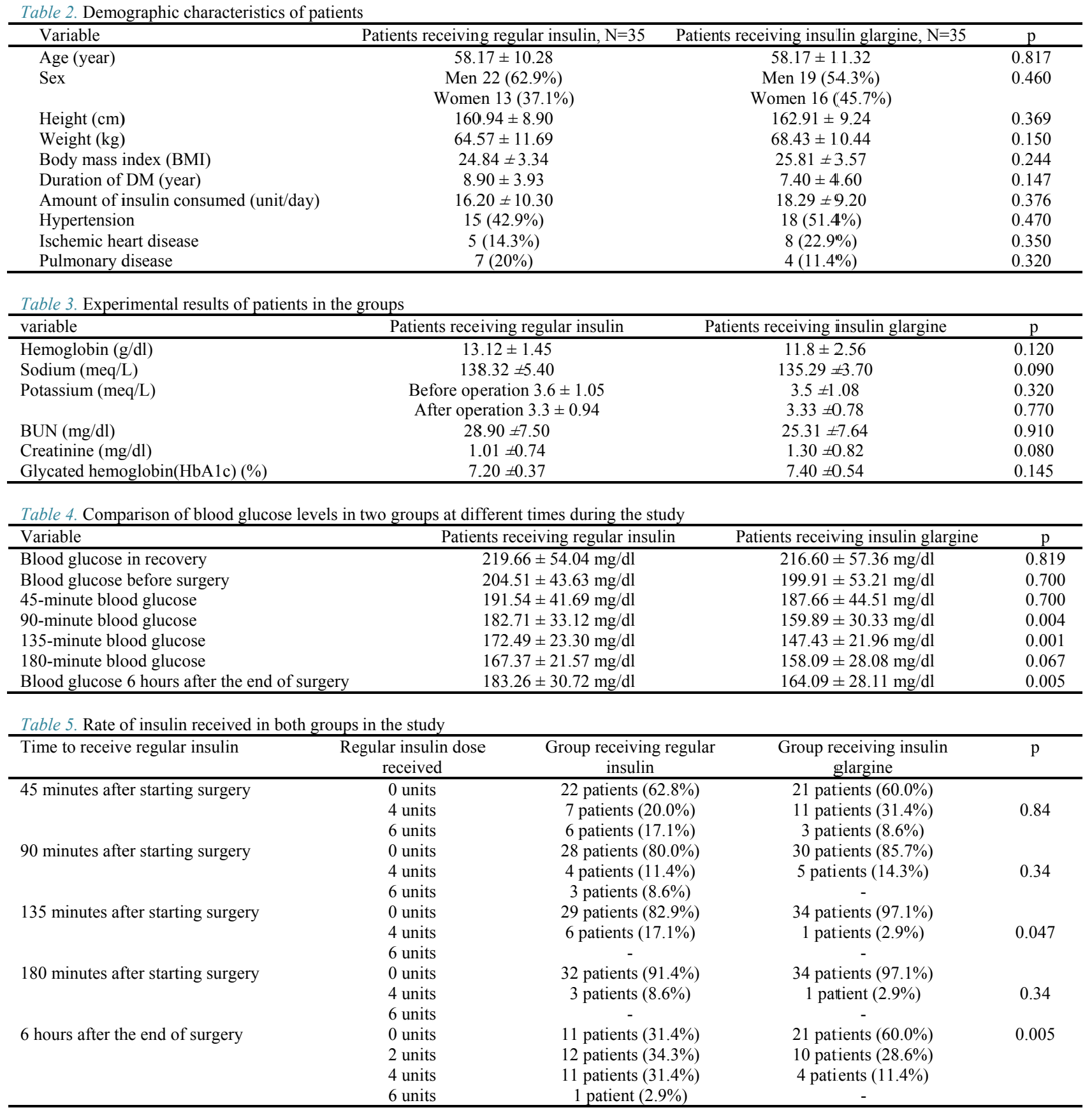


surgery and 135 minutes after starting surgery, regular insulin requirement during surgical procedure was significantly different from the group receiving insulin glargine (lanthus) ( $\mathrm{p}=0.047, \mathrm{p}=0.005)$.

The blood glucose variation along the time was compared by repeated measurement test as shown in Figure 2, where all of the 6-time variables was brought together. Figure 3 is based on the time of the blood glucose assessment between two groups and the time variables are shown separately by repeated measurement test. Results of the analysis of the repeated measurements showed that the use of insulin glargine was significantly more effective than regular insulin in the glycemic control among patients during eye surgery up to 6 hours after surgery (Fig. 2) $(\mathrm{p}=0.001)$.

\section{Discussion}

The results of this study indicated that the use of insulin glargine was significantly better than regular insulin in controlling the blood glucose in patients during eye surgery and up to 6 hours after surgery. In addition, the results showed that the blood glucose of patients 90 and 135

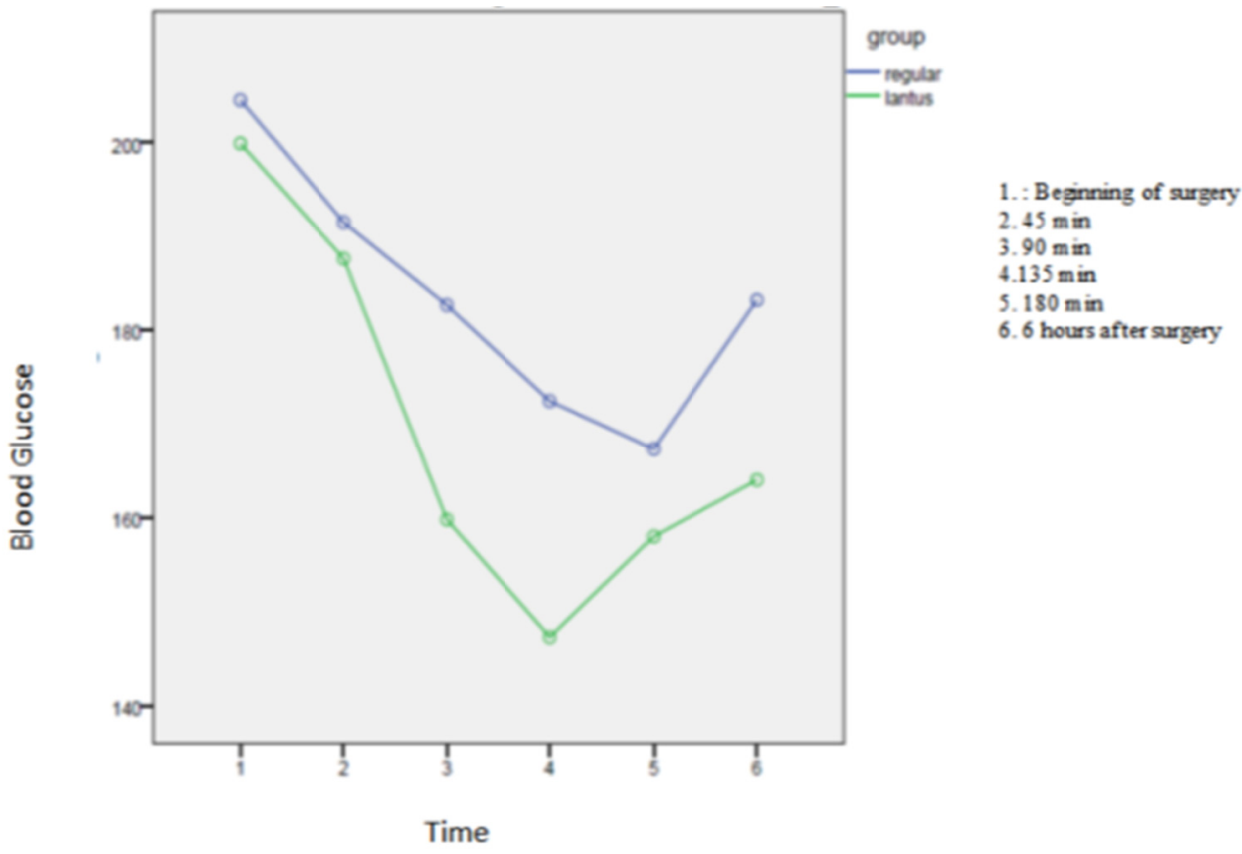

Fig. 2. Blood glucose variation in the two groups (repeated measures ANOVA)

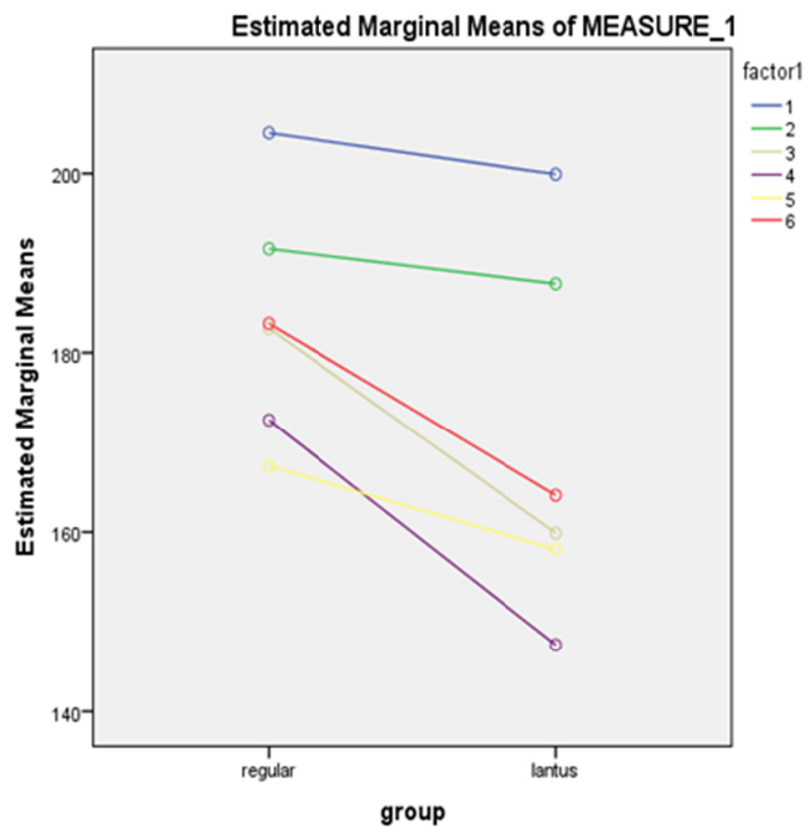

Factor 1: $\mathrm{BS}$ in beginning of surgery

Factor 2: $\mathrm{BS}$ in $45 \mathrm{~m}$ in

Factor 3: $\mathrm{BS}$ in $90 \mathrm{~m}$ in

Factor $4: \mathrm{BS}$ in $135 \mathrm{~m}$ in

Factor $5: \mathrm{BS}$ in $180 \mathrm{~m}$ in

Factor 6: 6 hours after surgery

Fig. 3. Blood glucose in separate times 
minutes after starting the surgery and 6 hours after the surgery were significantly different. None of the patients in the regular and glargine (Lanthus) group insulin experienced hypoglycemia during surgery and 6 hours after the surgery. 6 hours after surgery and 135 minutes after the start of surgery, the insulin requirement in the glargine (lanthus) group was significantly lower. Therefore, glycemic control in patients receiving glargine was better than those receiving regular insulin and the need for regular insulin doses after surgery was much lower.

In a study by Kang $\mathrm{H}$ et al. in femoral artery bypass to the popliteal artery and administration of insulin glargine at a rate of two-thirds of the total daily insulin dose, no case of hypoglycemia was reported (6). In the study by Aggarwal et al. on glycemic control (insulin infusion or receiving insulin glargine) in patients undergoing heart surgery during ICU admission, at a dose of $0.15 \mathrm{unit} / \mathrm{kg}$ (less than the dose used in the present study), no hypoglycemic event was reported (15). In the study by Silinskie et al., although hypoglycemia occurred in patients receiving regular and glargine insulin, it was similar in both groups (4). Nevertheless, hypoglycemia was reported in the study by Hagelberg et al. in patients receiving regular insulin (9), and in the study by Guillermo et al., hypoglycemia was more common in patients receiving glargine (7). Despite these studies, based on the results of the current study, hypoglycemia was not observed in any of the patients in the two groups. None of the patients in both regular and insulin glargine (lanthus) groups experienced hypoglycemia during surgery and 6 hours after the end of surgery. These inconsistencies necessitate more accurate investigations focusing on hypoglycemia in the future.

In a study by $\mathrm{G}$ Hemang et al. among patients undergoing coronary artery bypass graft (CABG) surgery (comparison of insulin infusion and glargine receiving with 1 unit $/ \mathrm{kg}$ before surgery), it was found that glucose control was better in the glargine group and the need for insulin was reduced during and after the operation. The higher dosage of insulin glargine could be involved in better results in this study in comparison to the present study (1). In the study by Aggarwal, which compared the two regular and glargine insulin, it was found that the mean glucose level was lower in the glargine group, but the difference was not significant. It should be noted that this study was performed in the postoperative period; however the current study was conducted during surgery and up to 6 hours after the operation (15). The results of the study by Rys et al. revealed that glargine provided insulin delivery with minimal fluctuation in the insulin levels and activity, and replaced insulin without a peak effect and regardless of the meal, and it was more effective in controlling blood glucose and lowering the risk of hypoglycemia (16). Based on the results of this study, it was also found that the use of insulin glargine was preferable in controlling the blood sugar of patients undergoing surgery.

In a few studies, investigation of the effect of glargine administration immediately after cardiac surgery has shown that the average daily glucose level in the glargine group was lower than that of the regular group and also reduced the burden of nursing without hypoglycemia (15,
17); in the present study, differences were not significant immediately after surgery.

The main limitation of the present study was the use of the sliding scale protocol to control the perioperative blood sugar level, despite the greater use of insulin infusion in controlling blood glucose, which can be due in part to a minor surgical procedure and lack of high fluctuations in blood sugar during operation.

\section{Conclusion}

It can be generally concluded that in blood glucose level above $150 \mathrm{mg} / \mathrm{dl}$ in patients with DM undergoing vitrectomy, controlling the perioperative blood sugar using glargine was more efficient and with less need for an extra dosage of regular insulin during and after surgery, and it can be used to control the perioperative blood glucose levels as well.

Given the results of this study, it is suggested that studies be carried out on the use of glargine in patients admitted to ICU and also with doses higher than 0.3 units $/ \mathrm{kg}$ during surgery. Similarly, more research is needed to assess the risk of hypoglycemia.

\section{Acknowledgments}

The authors would like to thank Iran University of Medical Sciences, Tehran, Iran, and also Rasoul Akram Hospital, and Dr. Ehsan Moosavi for editorial assistance.

\section{Conflict of Interests}

The authors declare that they have no competing interests.

\section{References}

1. Gandhi H, Sarvaia A, Malhotra A, Acharya H, Shah K, Rajavat JJAoca. Effects of glargine insulin on glycemic control in patients with diabetes mellitus type II undergoing off-pump coronary artery bypass graft. Ann Card Anaesth. 2018 Apr-Jun; 21(2):167-172.

2. Holden SE, Currie CJ. Endogenous hyperinsulinaemia and exogenous insulin: A common theme between atherosclerosis, increased cancer risk and other morbidities. Atherosclerosis. 2012 Feb;222(1):26-8

3. Ye X, Qi J, Yu D, Wu Y, Zhu Sh, Li Sh, et al. Pharmacological efficacy of FGF21 analogue, liraglutide and insulin glargine in treatment of type 2 diabetes. J Diabetes Compl. 2017 Apr;31(4):726734.

4. Silinskie KM, Kirshner R, Hite MS. Converting continuous insulin infusion to subcutaneous insulin glargine after cardiac surgery using percentage-based versus weight-based dosing: a pilot trial. Ann Pharmacother. 2013;47(1):20-8.

5. Barbie H. Implementing a Diabetes Optimization Protocol for Elective Surgery Patients. Walden University, ProQuest Dissertations Publishing 2020.

6. Russo N. Perioperative Glycemic Control. Anesthesiology Clin. 2012 Sep;30(3):445-66

7. Umpierrez GE, Smiley D, Jacobs S, Peng L, Temponi A, Mulligan P, et al. Randomized study of basal-bolus insulin therapy in the inpatient management of patients with type 2 diabetes undergoing general surgery (RABBIT 2 surgery). Diabetes Care. 2011;34(2):256-61.

8. Abdelmalak BB, Duncan AE, Bonilla A, Yang D, Parra-Sanchez I, Fergany A, et al. The intraoperative glycemic response to intravenous insulin during noncardiac surgery: a subanalysis of the DeLiT randomized trial. J Clin Anesth. 2016;29:19-29.

9. Shah NJ, Leis A, Kheterpal S, Englesbe MJ, Kumar SS. Association of intraoperative hyperglycemia and postoperative outcomes in patients undergoing non-cardiac surgery: a multicenter retrospective study. BMC Anesthesiol. 2020;20:106.

10. Yoo S, Lee HJ, Lee H, Ryu HG. Association between perioperative 
hyperglycemia or glucose variability and postoperative acute kidney injury after liver transplantation: a retrospective observational study.Anesth Analg 2017;124(1):35-41.

11. Hu GH, Duan L, Jiang M, Zhang CL, Duan YY. Wider intraoperative glycemic fluctuation increases risk of acute kidney injury after pediatric cardiac surgery. Ren Fail. 2018;40(1):611-7.

12. Hokka M, Egi M, Mizobuchi S. Glycated hemoglobin A1c level on the day of emergency surgery is a marker of premorbid glycemic control: a retrospective observational study. BMC Anesthesiol. 2018;18(1):180.

13. Kang Z, Huo JL, Zhai XJ. Effects of perioperative tight glycemic control on postoperative outcomes: a meta-analysis. Endocr Connect. 2018;7(12): R316-R327.

14. Lee YY, Lin UM, Leu WJ, Wu MY, Tseng JH, Hsu MT. Slidingscale insulin used for blood glucose control: a meta-analysis of randomized controlled trials. Metabolism. 2015 Sep ;64(9):1183-92.

15. Aggarwal A, Rawn J, Pendergrass M, Garg R. Long-acting subcutaneously administered insulin for glycemic control immediately after cardiac surgery. Endocr Pract. 2011;17(4):558-62.

16. Rys P, Wojciechowski P, Rogoz-Sitek A, Niesyczyński G, Lis J, Syta A. Systematic review and meta-analysis of randomized clinical trials comparing efficacy and safety outcomes of insulin glargine with NPH insulin, premixed insulin preparations or with insulin detemir in type 2 diabetes mellitus. Acta Diabetol. 2015 Aug;52(4):649-62

17. Aggarwal A, Rawn J, Pendergrass M, Garg R. Long-Acting Subcutaneously Administered Insulin for Glycemic Control Immediately After Cardiac Surgery. Endocr Pract. 2011 JulAug;17(4):558-62. 